\title{
Una aproximación a la concepción de ciencia en la contemporaneidad desde la perspectiva de la educación científica
}

\section{An approach to the conceiving science in contemporary settings from the scientific education perspective}

Esperanza Asencio Cabot ${ }^{1}$

\begin{abstract}
Resumen: El perfeccionamiento de los sistemas educativos en la etapa actual de desarrollo social, insiste en la necesidad de renovación de la educación científica, por su contribución a la formación de ciudadanos competentes que puedan actuar reflexivamente ante los crecientes cambios científicos y tecnológicos. Esa nueva visión de la educación científica implica el reconocimiento de la ciencia como una empresa humana en continua construcción, en el marco de un contexto social, político, económico e histórico, que condiciona su evolución. Precisamente, este artículo aborda el tema planteado y enfatiza en la clarificación de las ideas acerca de la concepción de ciencia y método científico, así como de las visiones deformadas que aún se trasmiten desde la enseñanza de la ciencia escolar.
\end{abstract}

Palabras clave: Educación científica. Concepción de ciencia. Método científico. Contemporaneidad.

\begin{abstract}
The improvement today of the educational systems emphasizes the need for scientific education, through its contribution to the formation of competent citizens, who can act reflectively on the increasing scientific and technology challenges. Scientific education's new vision recognizes science as a human enterprise in a continuous process of construction, framed by the social, political, economic, and historical context, which has led to its evolution. This paper deals with the this theme and it emphasizes the need for clarification of ideas about the conception of science and scientific methods, as well as identifying some false visions of the science, which are still transmitted in the teaching of science.
\end{abstract}

Keywords: Scientific education. Science conception. Scientific methods. Contemporaneity.

\footnotetext{
${ }^{1}$ Departamento de Matemática-Física, Facultad de Ciencias, Universidad de Ciencias Pedagógicas (UCP) "Félix Varela Morales", Carretera de Maleza y Circunvalación, Santa Clara, 50300, Villa Clara, Cuba.

E-mail: <easencio@ucp.vc.rimed.cu>
} 


\section{Introducción}

El perfeccionamiento de los sistemas educativos en la etapa actual de desarrollo social, centra su atención hacia el logro de una educación de calidad para todos a lo largo de la vida, enfatizando en el papel de la educación científica por su contribución a la formación de ciudadanos competentes que actúen reflexivamente en una sociedad marcada por los crecientes cambios científicos y tecnológicos.

$\mathrm{Al}$ respecto, en la Conferencia mundial celebrada en Budapest en 1999 se insistió en la necesidad de mejorar, reforzar y diversificar la educación científica, formal e informal, a todos los niveles y para todos los sectores, e integrar la ciencia a la cultura general con énfasis en su contribución a la formación de un pensamiento abierto y crítico, así como al mejoramiento de las habilidades de la población para sobrevivir ante los desafíos de la sociedad moderna; se consideró asimismo, que para que un país esté en condiciones de atender a las necesidades fundamentales de su población, la enseñanza de las ciencias y la tecnología es un imperativo estratégico. (UNESCO, 2005).

De manera similar, en la Conferencia Internacional para el desarrollo humano celebrada en la India en el 2001, se llegó a la conclusión de que el rasgo distintivo de la educación científica, tecnológica y matemática en su esfuerzo por animar la alfabetización científica y tecnológica es el énfasis en las necesidades sociales y en la necesidad de una ética de responsabilidad social en el desarrollo y aplicación de la ciencia y la tecnología. (UNESCO, 2005).

Muchas organizaciones internacionales, entre las que se destacan la Organización de las Naciones Unidas para la Educación, la Ciencia y la Cultura (UNESCO) y la Organización de Estados Iberoamericanos (OEI), entre otras, están trabajando en diversos programas y proyectos para promover una nueva visión de la educación científica que les permita a los alumnos comprender mejor el mundo en que viven y tomar sus propias decisiones (ORGANIZACIÓN DE ESTADOS IBEROAMERICANOS PARA LA EDUCACIÓN, LA CIENCIA Y LA CULTURA, 2010; UNESCO, 2005). En particular, el Proyecto Regional de Educación para América Latina y el Caribe, auspiciado por la UNESCO, enfatiza en la necesidad de que se asegure una educación científica de calidad, orientada al desarrollo sostenible y reconoce que "la formación científica y tecnológica de calidad para todos es un desafío pendiente ya que aún no ha sido incorporada de modo adecuado en todos los niveles educativos." (UNESCO, 2002, p. 11).

La oficina regional de la UNESCO para la América Latina y el Caribe está desarrollando desde hace varios años un programa para el mejoramiento de la educación científica en la región; en este programa se precisa que el objetivo primordial de la educación científica es formar a los alumnos para que sepan desenvolverse en un mundo impregnado por los avances científicos y tecnológicos, para que sean capaces de adoptar decisiones responsables y resolver los problemas cotidianos, por lo que se debe orientar la enseñanza de las ciencias hacia una ciencia para la vida y para el ciudadano tendiente a promover un futuro sostenible y posible. Este propio programa precisa que la nueva ciencia escolar debe promover el desarrollo de capacidades de valoración de la ciencia que les permita a los alumnos reconocerla como una empresa humana en continua construcción, con avances y retrocesos, en el marco de un contexto social, político, económico e histórico que condiciona su evolución. (UNESCO, 2005).

Son múltiples las investigaciones, innovaciones y experiencias pedagógicas de avanzada que trabajan por clarificar las ideas para la renovación de la educación científica en la época 
actual. Los estudios de las relaciones Ciencia-Tecnología-Sociedad (CTS) en el área educativa y las investigaciones en el campo de la Didáctica de las Ciencias han demostrado la importancia de reconocer la naturaleza de la ciencia y su dimensión social en la construcción de los conocimientos científicos en la enseñanza de las ciencias, y asimismo han aportado propuestas para favorecer el aprendizaje de los contenidos por los alumnos y su interés crítico hacia el papel de la ciencia y la tecnología en sus vidas, abordando las implicaciones sociales y éticas que el impacto tecnológico conlleva y los riesgos y amenazas impuestos por el propio avance científico-tecnológico. (GALVÃO; REIS; FREIRE, 2011; FURIÓ; GIL, 1999; GIL et al., 2005; HODSONA, 2003; KOLSTOE, 2000; MACEDO, 2006, 2008; McGINNIS; SIMMONS, 1999; NIEDA; MACEDO, 1997; RAMSEY, 1993; SANZ; LÓPEZ, 2012).

Sin embargo, en la práctica escolar aún se continúa transmitiendo una visión deformada de la naturaleza de la ciencia, de su objeto y métodos, de cómo se construyen y evolucionan los conocimientos científicos, ignorándose sus repercusiones sociales lo que en ocasiones provoca una actitud de rechazo hacia el área científica (CAMACHO, 2013; CASTRO; BEJARANO, 2013; GIL et al., 2005; MACEDO, 2008; MAIZTEGUI, 2002; RAVANAL; QUINTANILLA; LABARRERE, 2012; SANGIOGO et al., 2013; SILVA; CUNHA, 2012).

Precisamente, este artículo tiene como propósito ofrecer un acercamiento a la concepción de ciencia y método científico, desde la perspectiva de la educación científica, a fin de clarificar las ideas de lo que se entiende por ciencia, las formas fundamentales de sus manifestaciones, los rasgos esenciales que la caracterizan en la época actual, así como las visiones deformadas que aún subsisten en el pensamiento contemporáneo acerca de estos conceptos.

\section{Desarrollo}

\section{Algunas reflexiones en torno a la concepción de ciencia en la contemporaneidad}

El hombre en el proceso ininterrumpido de dominio y transformación de la realidad ha ido profundizando en sus conocimientos acerca de la naturaleza, la sociedad y el pensamiento. Con el proceso de división del trabajo y el surgimiento de las clases sociales aparecen las primeras reflexiones acerca del conocimiento científico, contenidas en aquellos momentos dentro del saber filosófico de la época, por lo que se reconoce por muchos estudiosos, a la filosofía como "ciencia de las ciencias" o "madre de las ciencias".

Con posterioridad, a través de procesos de diferenciación e integración del saber científico se fueron definiendo otros campos, apareciendo paulatinamente otras ciencias; estos procesos en la contemporaneidad han alcanzado un auge extraordinario, determinado por la aparición acelerada de nuevos conocimientos y en especial, por el desarrollo vertiginoso en el campo de la informática y las comunicaciones.

A lo largo de la historia de la humanidad, las ideas acerca de la ciencia han ido evolucionado en el tiempo, apareciendo diferentes modelos o visiones que en cierta forma han intentado integrar las formas de pensamiento de la época marcando etapas en su desarrollo. Así, baste mencionar las visiones de la ciencia acumulativa, el empirismo inductivista, la falsacionista de Popper, la paradigmática de Kuhn, los programas de Lakatos, entre otras; estas concepciones 
mencionadas, aun cuando pertenecen a épocas un tanto alejadas en el tiempo han tenido un arraigo tal, que todavía muchas de sus ideas persisten en el pensamiento contemporáneo, dando lugar a visiones deformadas de la naturaleza de la ciencia.

Una de las características del desarrollo científico de finales del siglo XX y principios del siglo XXI es el incremento de diferentes formas de integración horizontal (trabajo en equipos, multidisciplinariedad, interdisciplinariedad, transdisciplinariedad, entre otras) como recurso necesario para generar nuevos conocimientos y tecnologías; los conocimientos no están en la realidad, los construye el hombre; pero no el hombre aislado y ahistórico, sino el hombre en comunidad, el hombre en sociedad, que supone un diálogo, una relación entre razón y experiencia, entre teoría y empiria (NUÑEZ, 2005). Asimismo, es reconocida, la asociación directa entre la ciencia y el desarrollo económico con progreso social; la ciencia, al extender la frontera del conocimiento, posibilita la invención de nuevas técnicas y productos que posibilitan a su vez la aparición de nuevas actividades económicas, contribuyendo así a la creación de puestos de trabajo y al desarrollo social. (MOLTÓ, 2011).

Por otra parte, en el estado actual de las ciencias, se reconoce que la técnica participa tanto o más que la teoría en definir los objetos de estudio; así, en estas disciplinas, la observación y la experimentación se realizan mediante sofisticados instrumentos que median entre los fenómenos y su interpretación, de este modo, la técnica y la ciencia crean conjuntamente fenómenos nuevos. (MOLTÓ, 2011).

Corresponde seguidamente hacer un estudio de algunas ideas actuales que acerca de la definición de ciencia han aportado diferentes autores, a fin de poder analizar los elementos esenciales de dicho concepto.

Resulta difícil ofrecer una caracterización breve y precisa de lo que se entiende por ciencia. Por lo general, las definiciones dadas por los diferentes autores revelan, solo manifestaciones dispersas del fenómeno, que a veces resultan escurridizas e inalcanzables. (NUÑEZ, 2005). El término ciencia puede considerarse como polisemántico; su acepción depende de la óptica desde la cual se examina, de la época histórica y el contexto particular, así como de las referencias cosmovisivas sustentadas por cada especialista. (CASTELLANOS et al, 2005). Es por ello que el concepto de ciencia en nuestros días puede tomar diferentes significados. Entre las definiciones dadas por diferentes autores, vale hacer referencia a las que se presentan a continuación.

Ander Egg (1974), considera que la ciencia, es un conjunto de conocimientos racionales, ciertos o probables, obtenidos metódicamente, sistematizados y verificables, que hacen referencia a objetos de una misma naturaleza.

El colectivo de autores del Ministerio de Ciencia Tecnología y Medio Ambiente de Cuba (CITMA) considera que la ciencia, en su sentido más amplio se emplea para referirse al conocimiento sistematizado en cualquier campo, pero que suele aplicarse sobre todo a la organización de la experiencia sensorial objetivamente verificable. La búsqueda de conocimiento en ese contexto se conoce como 'ciencia pura', para distinguirla de la 'ciencia aplicada' (la búsqueda de usos prácticos del conocimiento científico) y de la tecnología, a través de la cual se llevan a cabo las aplicaciones. (CUBA, 2005).

Chávez (2005) destaca, que la ciencia es un saber que parte de lo objetivo y que se expresa subjetivamente en categorías, leyes y teorías, su alcance no es solo universal y atemporal, sino que posee un contenido histórico concreto, en tanto es un reflejo de un contexto 
socioeconómico determinado; es decir, se enlazan orgánicamente lo universal y lo particular en el saber científico. Además, la ciencia (saber) se aplica a la solución de los problemas de la vida mediante la técnica (saber hacer) que está estrechamente vinculada a ella y sin la cual resultaría una simple especulación. (CHÁVEZ, 2005).

Estas definiciones como se puede apreciar, revelan a la ciencia en su papel de conocimiento sistematizado expresado en categorías, leyes y teorías que reflejan las condiciones sociales y económicas de cada época. Sin embargo, otros autores, aportan nuevas ideas que pueden complementar el análisis del concepto objeto de estudio.

Así, Ruiz (2005), considera que la ciencia es la esfera de la actividad investigativa dirigida a la adquisición de nuevos conocimientos sobre la naturaleza, la sociedad y el pensamiento humano, que incluye todas las condiciones y elementos necesarios para ellos, entre los que se encuentran: los científicos, las instituciones científicas, los métodos de trabajo científico-investigativo, el aparato conceptual y categorial y el sistema de información científica.

Según el diccionario filosófico el concepto de ciencia incluye todas las condiciones y elementos necesarios para ello: los científicos, las instituciones científicas, los métodos de trabajo de investigación científica, el aparato conceptual y sus categorías, el sistema de información científica así como toda la suma de conocimientos existentes, que constituyen la premisa, el medio o el resultado de la producción científica. (ROSENTAL, 1984).

Por otra parte, Kröber (1986), entiende la ciencia no sólo como un sistema de conceptos, proposiciones, teorías e hipótesis, sino también, simultáneamente, como una forma específica de la actividad social dirigida a la producción, distribución y aplicación de los conocimientos acerca de las leyes objetivas de la naturaleza y la sociedad; aún más, la ciencia se nos presenta como una institución social, como un sistema de organizaciones científicas, cuya estructura y desarrollo se encuentran estrechamente vinculados con la economía, la política, los fenómenos culturales, con las necesidades y las posibilidades de la sociedad dada.

Estos autores mencionados, incorporan en sus definiciones algunos elementos en los que resulta importante reflexionar; entre estos se encuentran: la consideración de la ciencia como proceso al declararla como un tipo particular de actividad humana estrechamente vinculada con los métodos del trabajo científico-investigativo, así como acercan el concepto a la institución social enfatizando en la idea del trabajo colectivo y el papel de los científicos. Otras definiciones que se expondrán seguidamente muestran una visión más acabada del concepto de ciencia.

Así, Castellanos et al. (2005), expresan que la ciencia representa un complejo fenómeno de la vida espiritual humana, que penetra simultáneamente en la vida material, por cuanto se ha convertido en una fuerza productiva social directa, en las condiciones de la revolución científico-tecnológica contemporánea; como institución social, la ciencia se ocupa de un tipo particular de actividad humana, cuya singularidad y diferenciación se fundamentan en la cualidad del proceso mismo y de su producto o resultado, por cuanto representa un campo especial del conocimiento teórico, cuya finalidad es trascender las apariencias, explicando las leyes y mecanismos, los nexos y las propiedades que dimanan de la esencia.

Nuñez (2005), considera que la ciencia se puede analizar como sistema de conocimientos que modifica nuestra visión del mundo real y enriquece nuestro imaginario y nuestra cultura; se le puede comprender como proceso de investigación que permite obtener nuevos conocimientos, los que a su vez ofrecen posibilidades nuevas de manipulación de los fenómenos; es posible atender a sus impactos prácticos y productivos, caracterizándola como fuerza 
productiva que propicia la transformación del mundo y es fuente de riqueza; la ciencia también se nos presenta como una profesión debidamente institucionalizada, portadora de su propia cultura y con funciones sociales bien identificadas.

Estos especialistas citados con anterioridad, además de las facetas señalados por los demás autores, incorporan la visión de la ciencia como fuerza productiva, lo cual enriquece la definición y la acerca a las problemáticas más actuales del mundo de hoy.

El estudio realizado a partir de las definiciones presentadas y de otras no declaradas textualmente por limitaciones en el espacio de este material bibliográfico, no pretende arribar a una nueva definición de ciencia, sino llegar a establecer las formas de manifestación y los rasgos generales que la caracterizan en la época actual. Atendiendo a esta intención, se puede resumir que la ciencia se puede manifestar como:

- Cuerpo de conocimientos. Se conforma en un cuerpo de conocimientos sistematizados y coherentes, en constante revisión y reconstrucción, que se desarrollan con complejidad creciente y que se integran en determinados campos de la realidad objetiva (naturaleza, sociedad y pensamiento), los cuales se tipifican por un objeto de estudio, un aparato conceptual, categorial y legal con metodologías específicas y con problemas a resolver (inmediatos y perspectivos) que permiten describir, explicar, predecir y transformar la realidad.

- Proceso. Es un tipo especial de actividad humana, organizada, planificada y que tiene un fin consciente dirigido a resolver problemas y buscar nuevos conocimientos que enriquecen la teoría, empleando para ello diferentes métodos, procedimientos y técnicas.

- Institución social. Se estructura en una organización que desarrolla programas, proyectos, estudios, y otros, vinculada estrechamente con la política, la economía, la cultura, la educación, entre otros y que como parte de una misión colectiva integra a científicos y personal especializado atendiendo a los fines específicos que persigue.

- Fuerza productiva. Se relaciona directamente con la base económica de la formación económica social a la cual corresponde y constituye un factor de progreso social en las condiciones de la revolución científica-tecnológica contemporánea, así como una fuente de riqueza por el impacto de los beneficios que puede aportar a la sociedad.

Es importante destacar, que las formas de manifestación expresadas solo pueden reconocerse con un propósito analítico, a fin de explorar el fenómeno complejo que es la ciencia; las diferentes facetas a las que nos hemos referido se dan unidas en un todo integrado y por tanto no pueden verse aisladas ni separadas entre sí.

Por otra parte, el estudio realizado ha permitido reflexionar y precisar en algunos de los rasgos esenciales que son de vital trascendencia para una caracterización más completa del concepto en los momentos actuales; entre ellos se destacan los siguientes:

- Carácter socio-cultural. La ciencia en la época actual es fruto del trabajo colectivo y como construcción social es patrimonio de la sociedad, pues crea valores espirituales y materiales que deben estar dirigidos a la humanización del hombre y la transformación del mundo en aras del desarrollo humano, constituyendo una parte de la cultura. Su consideración como empresa colectiva en la época actual, marca una diferencia con la ciencia de siglos anteriores, la que presentaba un carácter más individualizado y aislado. Este carácter de actividad social en las actuales condiciones la vincula a las restantes formas de la actividad humana, por lo que los procesos de producción, difusión y aplicación de conocimientos propios de la actividad científica son inexplicables al margen de los intereses económicos, políticos, militares, entre otros. 
- Carácter histórico-concreto. La ciencia es reflejo de la época y está históricamente condicionada por el pensamiento dominante en un contexto político y socioeconómico determinado, de ahí su constante evolución en el tiempo, sus retrocesos y sucesivas rectificaciones resultado de la superación de múltiples obstáculos y cambios de paradigmas. Es por ello que la concepción de ciencia en nuestros días debe ser coherente con las problemáticas más actuales y contextuales condicionadas por la revolución científico-técnica contemporánea.

- Carácter ético. Por la propia naturaleza social de la ciencia, ella está comprometida con los valores, prioridades e intereses propios de la estructura y los agentes sociales, de ahí que esté involucrada y contaminada por ellos, por lo que no puede ser neutral. La selección de problemas y las estrategias para resolverlos deben tener en cuenta los intereses humanos más amplios; sin embargo, esta perspectiva no siempre está presente a la luz de los usos diversos, a veces antihumanos, que puede tener el conocimiento. Lo anterior reafirma la idea de la importancia en los momentos actuales, de ver en el desarrollo científico a los conocimientos y los valores indisolublemente unidos, y en especial destacar el compromiso social de la ciencia que la enfrenta a las posiciones de lucro y enriquecimiento personal que pueden engendrar sociedades donde el individualismo es un valor dominante.

- Carácter complejo. La ciencia en la sociedad actual constituye un fenómeno social complejo, si se tiene en cuenta las diversas manifestaciones que puede adoptar, las cuales se relacionan con diferentes niveles de la Formación Económico Social (FES) en un contexto particular. De esta forma, su papel como fuerza productiva la sitúa en la base económica, lo cual determina un sistema de interrelaciones con los demás elementos que integran el modo de producción de una sociedad dada. Por otra parte, la ciencia se ubica en la superestructura de la FES en su manifestación como cuerpo de conocimientos, lo que implica interacciones diversas con aspectos políticos, jurídicos, filosóficos, religiosos, culturales, entre otros; asimismo queda ubicada también en la superestructura en su papel de institución, determinando interacciones particulares con otras instituciones sociales. Una visión integral considerando los elementos citados en los diferentes niveles estructurales de la FES (que a su vez tienen una repercusión recíproca y una dialéctica particular entre ellos) y teniendo en cuenta además, que en la estructura social, la comunidad científica también interactúa con otros grupos y clases sociales, permite advertir en toda su magnitud el complejo entramado de interrelaciones vinculados a la ciencia en la época actual.

Hasta aquí se ha realizado una aproximación al concepto de ciencia en la época actual, sin embargo en el pensamiento contemporáneo aún persisten ideas que presentan imágenes deformadas de dicho concepto, que han tenido una repercusión negativa en el aprendizaje de las ciencias. Entre esas ideas se destacan las visiones: descontextualizadas, individualistas y elitistas, empíricas y ateóricas, rígidas e infalible, aproblemáticas, ahistóricas, exclusivamente analíticas y acumulativas (GIL et al., 2005).

Estas concepciones aparecen asociadas entre sí, dando como resultado a imágenes ingenuas, distorsionadas, empobrecidas y alejadas de la realidad, que se ha ido trasmitiendo de forma explícita o implícita, pasando a ser socialmente difundidas y aceptadas. Estas visiones en el campo educativo dan lugar a errores y simplismos que generan el fracaso y el desinterés hacia el aprendizaje de las ciencias y hacia la ciencia misma. (GIL et al., 2005). Es por ello que la superación de esas visiones deformadas de la ciencia debe considerarse como un requisito ineludible para la renovación de la educación científica. 
Hasta aquí, se ha pretendido dar una panorámica general a modo de clarificar algunas ideas importantes en relación con lo que se entiende por ciencia en nuestros días. Se considera oportuno también reflexionar acerca del término método científico, muy vinculado tradicionalmente a la ciencia y el cual en los momentos actuales suscita opiniones controvertidas en relación con su empleo.

\section{El método científico o métodos de la ciencia: una polémica actual}

La palabra método, según el diccionario Larrouse (1968), procede del griego methodos (de meta, con y odos, vía) de ahí que se relacione con el modo razonado de obrar. Según el diccionario filosófico, el método es la manera de abordar la realidad, de estudiar los fenómenos de naturaleza y la sociedad. (ROSENTAL, 1984). La enciclopedia filosófica lo define como la forma de asimilación teórica y práctica de la realidad que parte de las regularidades del movimiento del objeto estudiado o como el sistema de principios reguladores de la actividad transformadora práctica, cognoscitiva y teórica. (MARTínEZ, 2005).

En la ciencia, el método se manifiesta a través de la forma de investigación y disposición del material de estudio, mediante la solución de tareas de carácter teórico, práctico, cognoscitivo y otros (MARTÍNEZ, 2005), por lo que resulta indiscutible el estrecho vínculo entre ciencia y método. Esta unión indisoluble, manifestada desde los albores de la ciencia, es lo que explica el empleo del término método científico desde esa época, aunque en realidad no se puede hablar en un sentido estricto de una metodología del pensamiento científico en esos primeros momentos del surgimiento de la ciencia. (FEDOSÉEV, 1978).

$\mathrm{Al}$ igual que la ciencia, el método científico tiene un carácter histórico-concreto, por lo que a lo largo de la historia de la humanidad las ideas acerca de su significado han ido evolucionando con el tiempo y el contexto donde las mismas se han generado. Sin embargo, en este devenir del desarrollo científico las concepciones del método científico, vinculada a enfoques de la ciencia acumulativa e inductiva, aun cuando pertenecen a épocas un tanto alejadas en el tiempo, han tenido un arraigo tal, que todavía muchas de sus ideas persisten en la actualidad. Esto ha dado lugar, a visiones desactualizadas de la naturaleza del método científico que lo alejan de la concepción de la ciencia en la etapa actual de desarrollo social, pero que subyacen en el pensamiento contemporáneo.

Esas visiones del método científico lo identifican con un algoritmo infalible de validez universal, conformado por unas reglas rígidas y perfectamente ordenadas, en una secuencia de etapas definidas, independientes del contexto, en las que las observaciones y los experimentos rigurosos juegan un papel destacado, garantizando la exactitud y objetividad de los resultados; estas ideas expresadas han provocado una posición de rechazo en algunos especialistas al empleo del término método científico. (GIL et al., 2005; NIEDA; MACEDO, 1997; VALDÉS; VALDÉS, 1999). Sin embargo otros autores, mantienen su aceptación por dicho término y han logrado en sus definiciones una aproximación coherente con la concepción actual de ciencia, en un sentido estratégico y desde una perspectiva holística.

Así, García (1999, citado por CASTELLANOS et al., 2005), asume el método científico como la estrategia que organiza y orienta la actividad científica, encaminada a la obtención de un nuevo conocimiento científico que transforme la realidad, mientras que, Fernández et al. (2005) 
consideran al método científico, como el camino general para penetrar en el conocimiento de una porción del mundo natural o social, que ha de encontrar formas singulares de aplicación para adecuarse a la realidad del objeto, asumiendo que éste existe en espacios y tiempos que representan auténticas variables intervinientes de forma activa en la cualidad y expresión de los hechos y procesos estudiados.

Por otra parte, Castellanos et al. (2005) insisten en que el método científico es la estrategia general del pensamiento científico-teórico, que orienta y regula conscientemente el proceso dialéctico de construcción del conocimiento por el sujeto cognoscente en correspondencia con las particularidades del objeto, con la finalidad de aprehender su esencia y transformar la realidad; el método sigue el camino dialéctico del conocimiento y abarca el proceso investigativo en su totalidad, en todos sus momentos y eslabones.

Martínez (2005) plantea que en la esencia el método científico viene a ser una teoría práctica, dirigida a la actividad misma de la investigación, o lo que es lo mismo, la teoría verificada por la práctica y utilizada como principio regulador del proceso de conocimiento; las reglas de acción práctica del hombre se subordinan a la lógica objetiva, por ello el método no sólo se encuentra en unión estrecha con la teoría, sino que cumple la función de principio unificador entre la teoría y la práctica.

Según se ha advertido en los planteamientos anteriores, con relación al método científico, hay autores que tienden a comprenderlo en un sentido estrecho, como ejecución ordenada de un conjunto de pasos y prescripciones que suelen devenir recetarios formales, pero existe en la actualidad un consenso amplio en cuanto a la necesidad de abordarlo como una estrategia global de enfrentamiento al conocimiento científico del mundo, desde una visión holística del proceso investigativo, desarrollado alrededor del método como pauta flexible y amplia que cada investigador adapta en función de su campo de estudio particular y de otras variables contextuales y coyunturales. (FERNÁNDEZ et al., 2005).

En resumen, respetando las posiciones divergentes de los diferentes autores en cuanto a la aceptación o rechazo del empleo del término método científico, se considera que la cuestión medular es reconocer la necesidad en el trabajo científico, de una proyección estratégica global (independientemente de la denominación con la cual se identifique) que contribuya a enfrentar el conocimiento científico del mundo, para penetrar y transformar la realidad objetiva, la cual:

- organiza, orienta y regula de forma consciente la actividad científica.

- constituye una pauta flexible y amplia.

- se adapta a campos particulares y variables temporales y contextuales.

- emplea diversos métodos, procedimientos y técnicas.

- se encuentra interrelacionada con un cuerpo teórico determinado.

- toma en cuenta factores subjetivos y posiciones éticas.

\section{Conclusiones}

En este artículo ha sido abordada la concepción de ciencia con la intención de clarificar las ideas acerca de su naturaleza, las que deben ser incorporadas a la renovación de la educación científica en la contemporaneidad. 
El análisis realizado permitió llegar a establecer las formas fundamentales de manifestación de la ciencia en la época actual; así la misma puede ser considerada como: cuerpo de conocimientos, proceso, institución social y fuerza productiva. Asimismo, en el trabajo se reflexionó y precisó en algunos rasgos esenciales que caracterizan la ciencia en la actualidad, entre los que se destacan su carácter socio-cultural, histórico-concreto, ético y complejo.

Por otra parte, se profundizó en las ideas acerca del método científico, las cuales han evolucionado a lo largo de la historia de la humanidad y que en nuestros días se considera como una estrategia global de enfrentamiento al conocimiento del mundo encaminado a penetrar y transformar la realidad. Por último, en el artículo se analizan brevemente las ideas que aún persisten en el pensamiento contemporáneo, que presentan imágenes deformadas de la ciencia y la actividad científica y que son socialmente difundidas y aceptadas.

En sentido general, se considera que este trabajo puede contribuir a clarificar las ideas de los docentes relacionadas con la naturaleza de la ciencia y el método científico y a reconocer la importancia del tratamiento de las mismas desde la ciencia escolar como un aspecto esencial en el perfeccionamiento de la educación científica en la actualidad.

\section{Referencias}

CAMACHO, J. P. Concepciones sobre ciencia y género en el profesorado de química: aproximaciones desde un estudio colectivo de casos. Ciência \& Educação, Bauru, v. 19, n. 2, p. 323-338, 2013.

Disponible en: <http://dx.doi.org/10.1590/S1516-73132013000200007>. Visitado el: 10 Ago. 2013.

CASTELLANOS, B. et al. Esquema conceptual, referencial y operativo sobre la investigación educativa. La Habana: Editorial Pueblo y Educación, 2005.

CASTRO, D. R.; BEJARANO, N. R. R. Os conhecimentos alternativos e científicos na área de ciências naturais: uma revisão a partir da literatura internacional. Ciência \& Educação, Bauru, v. 19, n. 1, p. 55-71, 2013. Disponible en: <http://dx.doi.org/10.1590/S1516-73132013000100002>. Visitado el: 10 Ago. 2013.

CHÁVEZ, J. Acercamiento necesario a la pedagogía general. La Habana: Editorial Pueblo y Educación, 2005.

CUBA. Ministerio de Ciencia Tecnología y Medio Ambiente. Ciencia y revolución. La Habana: MINED, 2005.

EGG, A. Introducción a las técnicas de investigación social. Buenos Aires: Humanites, 1974.

FEDOSÉEV, P. N. Metodología del conocimiento científico: Academia de Ciencias de la URSS y Cuba. La Habana: Editorial de Ciencias Sociales, 1978.

FERNÁNDEZ, A. M. et al. La investigación educativa desde un enfoque dialéctico. La Habana: MINED, 2005. 
Una aproximación a la concepción de ciencia...

FURIÓ, C.; GIL, D. Hacia la formulación de programas eficaces en la formación continuada del profesorado de ciencias. In: SANCHÉZ JIMÉNEZ, J. M.; OÑORBE DE TORRE, A. M.; BUSTAMANTE GUTIÉRREZ, I. (Coord.). Educación científica. Madrid: Servicio de Publicaciones Universidad de Alcalá, 1999. p. 129-148.

GALVÃO, C.; REIS, P.; FREIRE, S. A discussão de controvérsias sociocientíficas na formação de profesores. Ciência \& Educação, Bauru, v. 7, n. 3, p. 505-522, 2011. Disponible en: < http://dx.doi. org/10.1590/S1516-73132011000300001>. Visitado el: 10 Ago. 2013.

GIL, D. et al. ¿Cómo promover el interés por la cultura científica? Santiago de Chile: Andros Impresores, 2005.

HODSONA, D. Time for action: science education for an alternative future. International Journal of Science Education, London, v. 25, n. 6, p. 645-670, 2003. Disponible en: <http://dx.doi. org/10.1080/09500690305021>. Visitado el: 17 Jul. 2014.

KOLSTOE, S. D. Consensus projects: teaching science for citizenship. International Journal of Science Education, London, v. 22, n. 6, p. 645-664, 2000. Disponible en: <http://dx.doi. org/10.1080/095006900289714>. Visitado el: 17 Jul. 2014.

KRÖBER, G. Acerca de las relaciones entre la historia y la teoría del desarrollo de las ciencias.

Revista Cubana de Ciencias Sociales, La Habana, v. 4, n. 10, p. 27-32, 1986.

LARROUSE. Diccionario ilustrado. La Habana: Instituto del Libro, 1968.

MACEDO, B. Cultura y formación científica. La Habana: MINED, 2008.

MACEDO, B. Habilidades para la vida: contribución desde la educación científica en el marco de la década de la educación para el desarrollo sostenible. La Habana: MINED, 2006.

MAIZTEGUI, A. et al. Papel de la tecnología en la educación científica: una dimensión olvidada.

Revista Iberoamericana de Educación, Madrid, n. 28, p. 129-155, 2002. Disponible en: <http:// www.rieoei.org/rie28f.htm> .Visitado el: 10 Ago. 2013.

MARTÍNEZ, M. Naturaleza del método científico. La Habana: MINED, 2005.

McGINNIS, J. R.; SIMMONS, P. Teachers' perspectives of teaching science-technology-society in local cultures: a sociocultural analysis. Science Education, Hoboken, v. 83, n. 2, p.179-211, 1999.

MOLTÓ, M. Reseña de "La ciencia: entre valores modernos y posmodernidad de Gilbert Hottois". Revista Iberoamericana de Ciencia, Tecnología y Sociedad, Buenos Aires, v. 7, n. 19, 2011. Disponible en: <http://www.redalyc.org/pdf/924/92422643017.pdf>. Visitado el: 15 Ene. 2012.

NIEDA, J.; MACEDO, B. Un currículo científico para estudiantes de 11 a 14 años. Madrid: OIE, 1997.

NUÑEZ, J. La ciencia y la tecnología como procesos sociales. La Habana: MINED, 2005.

ORGANIZACIÓN DE ESTADOS IBEROAMERICANOS PARA LA EDUCACIÓN, LA

CIENCIA Y LA CULTURA. 2021 metas educativas: la educación que queremos para la generación de los bicentenarios - documento final. Madrid: OEI, 2010. Disponible en: <http://www.oei.es/ metas2021.pdf >. Visitado el: 03 Feb. 2012.

RAMSEY, J. The science education reform movement: implications for social responsability. Science Education, Hoboken, v. 77, n. 2, p. 235-258, 1993. 
RAVANAL, E.; QUINTANILLA, M.; LABARRERE, A. Concepciones epistemológicas del profesorado de biología en ejercicio sobre la enseñanza de la biología. Ciência \& Educação, Bauru, v. 18, n. 4, p. 875-895, 2012. Disponible en: <http://dx.doi.org/10.1590/S151673132012000400009>. Visitado el: 10 Ago. 2013.

ROSENTAL, M. Diccionario filosófico. La Habana: Editorial Ciencias Sociales, 1984.

RUIZ, A. Introducción a la investigación en la educación. La Habana: MINED, 2005.

SANGIOGO, F. A. et al. Pressupostos epistemológicos que balizam a Situação de Estudo: algumas implicações ao processo de ensino e à formação docente. Ciência \& Educação, Bauru, v. 19, n. 1, p. 35-54, 2013. Disponible en: <http://dx.doi.org/10.1590/S1516-73132013000100004>. Visitado el: 10 Ago. 2013.

SANZ, N.; LÓPEZ, J. A. Cultura científica para la educación del siglo XXI. Revista Iberoamericana de Educación, Madrid, n. 58, p. 35-59, 2012. Disponible en: < http://www.rieoei.org/rie58a02.pdf >. Visitado el: 04 Mayo 2012.

SILVA, F.; CUNHA, A. M. Método científico e prática docente: as representações sociais de professores de ciências do ensino fundamental. Ciência \& Educação, Bauru, v. 18, n. 1, p. 41-54, 2012. Disponible en: <http://dx.doi.org/10.1590/S1516-73132012000100003>. Visitado el: 10 Ago. 2013.

UNESCO. Proyecto regional de educación científica. Santiago de Chile: UNESCO, 2005.

UNESCO. Proyecto regional de educación para América Latina y el Caribe (PRELAC). Santiago de Chile: UNESCO, 2002.

VALDÉS, P.; VALDÉS, R. Enseñanza-aprendizaje de las ciencias en secundaria básica. La Habana: PROMET Editorial, 1999. 